Journal for ImmunoTherapy of Cancer

\title{
Newly developed pseudogout arthritis after therapy with MAGE-A4 directed TCR $T$ cells responded to treatment with tocilizumab
}

\author{
Sang T. Kim, ${ }^{1}$ Jean Tayar, ${ }^{1}$ Siqing Fu, ${ }^{2}$ Danxia Ke, ${ }^{2}$ Elliot Norry, ${ }^{3}$ Amy Sun, ${ }^{3}$ \\ Juli Miller, ${ }^{3}$ David S. Hong ${ }^{2}$
}

To cite: Kim ST, Tayar J, Fu S, et al. Newly developed pseudogout arthritis after therapy with MAGE-A4 directed TCR T cells responded to treatment with tocilizumab. Journal for ImmunoTherapy of Cancer 2021;9:e002716. doi:10.1136/jitc-2021-002716

Accepted 28 May 2021
Check for updates

(C) Author(s) (or their employer(s)) 2021. Re-use permitted under CC BY-NC. No commercial re-use. See rights and permissions. Published by BMJ.

${ }^{1}$ General Internal Medicine, The University of Texas MD Anderson Cancer Center, Houston, Texas, USA

${ }^{2}$ Department of Investigational Cancer Therapeutics, The University of Texas MD Anderson Cancer Center, Houston, Texas, USA

${ }^{3}$ Adaptimmune Therapeutics plc, Philadelphia, Pennsylvania, USA

Correspondence to

Dr Sang T. Kim;

stkim@mdanderson.org

\section{ABSTRACT}

With durable cancer responses, genetically modified cell therapies are being implemented in various cancers. However, these immune effector cell therapies can cause toxicities, including cytokine release syndrome (CRS) and immune effector cell-associated neurotoxicity syndrome (ICANS). Pseudogout arthritis is an inflammatory arthritis induced by deposition of calcium pyrophosphate dihydrate crystals. Here, we report a case of pseudogout arthritis in a patient treated with MAGE-A4 directed T cell receptor $T$ cells, for fallopian tube cancer. The patient developed CRS and ICANS 7 days after infusion of the T cells. Concurrently, the patient newly developed sudden onset of left knee arthritis. Synovial fluid analyses revealed the presence of calcium pyrophosphate dihydrate crystal. Notably, the pseudogout arthritis was resolved with tocilizumab, which was administered for the treatment of CRS and ICANS. Immunoprofiling of the synovial fluid showed that the proportion of inflammatory interleukin 17 (IL-17)-producing $\mathrm{CD}^{+} \mathrm{T}$ (Th17) cells and amount of IL-6 were notably increased, suggesting a potential role of Th17 cells in pseudogout arthritis after T-cell therapy. To the best of our knowledge, this is the first reported case of pseudogout arthritis after cell therapy. Clinicians, especially hematologists, oncologists and rheumatologists, should be aware that pseudogout arthritis can be associated with CRS/ICANS.

\section{INTRODUCTION}

Immune effector cell (IEC) therapies, including chimeric antigen receptor (CAR) and $\mathrm{T}$ cell receptor (TCR) therapies, have opened a new chapter in cancer therapeutics. ${ }^{1}$ Anti-CD19 CAR-T cell therapies (such as axicabtagene ciloleucel, tisagenlecleucel, brexucabtagene autoleucel, and lisocabtagene maraleucel) have shown clinical benefits and have recently been approved by the U.S. Food and Drug Administration for diffuse large B cell lymphoma, mantle cell lymphoma, and acute lymphoblastic leukemia. However, IEC therapy can cause toxicities including cytokine release syndrome (CRS) and immune effector cell-associated neurotoxicity syndrome (ICANS). ${ }^{1}$ CRS is the most common toxicity of IEC therapy, and its clinical manifestations include fever, tachycardia, hypotension, dyspnea, and organ dysfunctions. Inflammatory cytokines secreted from activated $\mathrm{T}$ cells and bystander immune cells including interleukin (IL)-6, interferon gamma (IFN $\gamma$ ), and granulocyte-macrophage colony stimulating factor (GM-CSF) are thought to play a critical role in CRS. ${ }^{2}$ ICANS is another toxicity of IEC therapy. Patients with ICANS have various neurologic symptoms including delirium, hallucination, cognitive dysfunction, and/or memory deficits, seizures, ataxia, and encephalopathy. ${ }^{1}$ The underlying pathogenesis of ICANS is elusive. Treatment for IEC therapy toxicities includes supportive care and immunosuppressants such as tocilizumab, an IL-6 receptor blocker). ${ }^{1}$ Of note, due to concerns of IEC suppression, steroids are frequently reserved as a second-line therapy for CRS refractory to tocilizumab. ${ }^{134}$

Pseudogout, induced by the deposition of calcium pyrophosphate dihydrate (CPPD) crystal, is an autoinflammatory disorder. ${ }^{5}$ The innate immune system and inflammasomes are known to play an essential role in its pathogenesis. ${ }^{5}$ Here, we report the first case of newly developed, crystal-proven pseudogout arthritis after infusion of MAGE A4 directed TCR T cells. The patient developed pseudogout arthritis along with CRS and ICANS. Interestingly, tocilizumab, which was used to treat the CRS and ICANS, also worked for the pseudogout arthritis. In parallel, we performed immunoprofiling of the synovial fluid obtained at the pseudogout flare.

\section{Case presentation}

The patient was a 78-year-old woman with a history of hypertension. The patient had 


\begin{tabular}{lll}
\hline \multicolumn{2}{l}{ Table 1} & Laboratory \\
\hline Test & Reference range & Result \\
\hline Calcium & $8.4-10.2(\mathrm{mg} / \mathrm{dL})$ & 8.7 \\
Phosphorus & $2.5-4.5(\mathrm{mg} / \mathrm{dL})$ & 3.5 \\
Magnesium & $1.6-2.6(\mathrm{mg} / \mathrm{dL})$ & 1.9 \\
\hline TSH & $0.27-4.20(\mathrm{mcunit} / \mathrm{mL})$ & 1.00 \\
Hemoglobin & $12.0-16.0(\mathrm{gm} / \mathrm{dL})$ & 8.2 \\
AST & $0-32(\mathrm{U} / \mathrm{L})$ & 21 \\
ALT & $0-33(\mathrm{U} / \mathrm{L})$ & 17 \\
ALP & $35-104(\mathrm{U} / \mathrm{L})$ & 63 \\
\hline Total bilirubin & $0-1.2(\mathrm{mg} / \mathrm{dL})$ & 0.7 \\
Direct bilirubin & $0-0.3(\mathrm{mg} / \mathrm{dL})$ & 0.1 \\
\hline
\end{tabular}

ALP, alkaline phosphatase; ALT, alanine aminotransferase; AST, aspartate aminotransferase; $\mathrm{TSH}$, thyroid stimulating hormone.

been well until early 2015, when she started to have nausea and vomiting. Workup revealed high-grade serous carcinoma of the right fallopian tube. The patient underwent neo-adjuvant chemotherapy with carboplatin plus paclitaxel followed by total abdominal hysterectomy and bilateral salpingo-oophorectomy. The patient received adjuvant chemotherapy with carboplatin plus taxotere. However, 1 year later, the tumor recurred and the patient underwent chemotherapy with carboplatin plus doxorubicin. The tumor response was inadequate, so the patient enrolled in a clinical trial and received MAGE-A4 directed TCR T cells. Seven days after the infusion, the patient developed a fever $\left(38.6^{\circ} \mathrm{C}\right)$, nausea, and difficulty writing and calculating. She also developed sudden-onset pain and swelling in her left knee. No similar episodes of joint pain and/or swelling had occurred in the past. Diagnostic arthrocentesis was performed. Synovial fluid analysis showed a white cell count within the inflammatory range $(12714 / \mu \mathrm{L})$ and numerous intracellular and extracellular CPPD crystals. Gram stain and culture were negative. Serum IL-6 was elevated at $260 \mathrm{pg} / \mathrm{mL}$ (normal range: $0-5 \mathrm{pg} / \mathrm{mL}$ ). Secondary causes of pseudogout arthritis, including hypothyroidism, hyperparathyroidism, hypomagenesemia, hypophosphatemia, and hemochromatosis, ${ }^{6}$ were ruled out either clinically or by laboratory tests (table 1). CT of the head was unremarkable, but brain MRI revealed hyperintensity on the posterior cerebral sulci. The patient was diagnosed with CRS and ICANS. The grade of CRS and ICANS were 1 and 2, respectively. For CRS and ICANS, the patient received $8 \mathrm{mg} / \mathrm{kg}$ of tocilizumab intravenously, and her symptoms, including arthritis, resolved over 3 days.

\section{MATERIALS AND METHODS Isolation of cells}

After the diagnostic arthrocentesis, residual synovial fluid of the left knee was collected. Synovial fluid samples were incubated with $10 \mathrm{IU}$ collagenase III
(Sigma, Cat No: H3506) at $37^{\circ} \mathrm{C}$ for $30 \mathrm{~min}$. After incubation, the sample was centrifuged at $500 \mathrm{G}$ for $10 \mathrm{~min}$ and the synovial fluid supernatant was collected. The remaining cells were washed with phosphate-buffered saline (Gibco) and cryopreserved in the presence of 90\% fetal bovine serum (Gibco, Cat No: 16140071) and $10 \%$ dimethyl sulfonoxide (Sigma, Cat No: D2650).

\section{Flow cytometry}

Cryopreserved synovial fluid cells were thawed, washed, and stained with flow cytometry antibodies. For intracellular cytokine staining, cells were stimulated for 4 hours in the presence of cell stimulation cocktail containing phorbol 12-myristate-13-acetate, ionomycin, and brefeldin A (Biolegend, Cat No: 423303). Subsequently, cells were stained to detect surface molecules, fixed (BD CytoFix/CytoPerm, Cat No: 51-2090KZ) and permeablized (BD PERM/Wash solution, Cat No: 51-2091KZ). After permeablization, the cells were stained to detect intracellular molecules. Flow cytometry data were acquired by BD LSR II FORTESSA X-20 and analyzed with FlowJo software (TreeStar, CA). Following antibodies were used for the flow cytometry; LIVE/DEAD Zombie Aqua (BioLegend), anti-CD19 PE (HIB19, BioLegend), anti-CD3 PerCP/Cyanine 5.5 (SK7, BioLegend), anti-TCR gamma/delta Brilliant Violet 421 (B1, BioLegend), anti-CD45RA Brilliant Violet 785 (HI100, BioLegend), anti-CD56 FITC (HCD56, BioLegend), anti-CD19 Brilliant Violet 785 (HIB19, BioLegend), anti-CCR7 PE-Cy7 (G043H7, BioLegend), anti-CD4 BUV395 (SK3, BD Horizon), anti-CD8 Alexa Fluor 700 (HIT8a, BioLegend), anti-CD25 FITC (BC96, BioLegend), anti-C-X-C chemokine receptor type 5 (CXCR5) APC (J25D4, BioLegend), anti-CD127 Alexa Fluor 700 (A019D5, BioLegend), anti-IL-4 Brilliant Violet 421 (MP4-25D2, BioLegend), anti-IL-21 PE (3A3-N2.1, BD Horizon), anti-IFN $\gamma$ PE/Dazzle 594 (4S.B3, BioLegend), anti-IL-17A PE-Cy7 (BL168, BioLegend).

\section{Enumeration of synovial immune cells}

To enumerate major immune cell subsets, we adapted and modified the gating strategy which we previously published. ${ }^{7}$ We calculated proportions of $\mathrm{CD} 4^{+} \mathrm{T}$ cell subsets including CD45RA ${ }^{+}$naïve, regulatory $\mathrm{T}$ cells (Tregs; CD25 ${ }^{\text {hi }} \mathrm{CD} 127^{\text {lo }}$ ), CXCR5 expressing follicular helper $\mathrm{T}$ cells, a distinct $\mathrm{CD} 4^{+} \mathrm{T}$ cell subset helping B cells ${ }^{8}$ and CD45RA CXCR5 effector cells. We also enumerated $\mathrm{CD} 4^{+} \mathrm{T}$ cells producing effector cytokines including IFN $\gamma$, IL-4, IL-17, and IL-21.

\section{Cytokine measurement}

The levels of IFN $\gamma$, tumor necrosis factor alpha, GM-CSF, IL-1 $\beta$, IL-2, IL-4, IL-6, IL-10, IL-17A, IL-21, and IL-22 were measured by multiplex ELISA techniques using commercially available kits (U-Plex Th17 

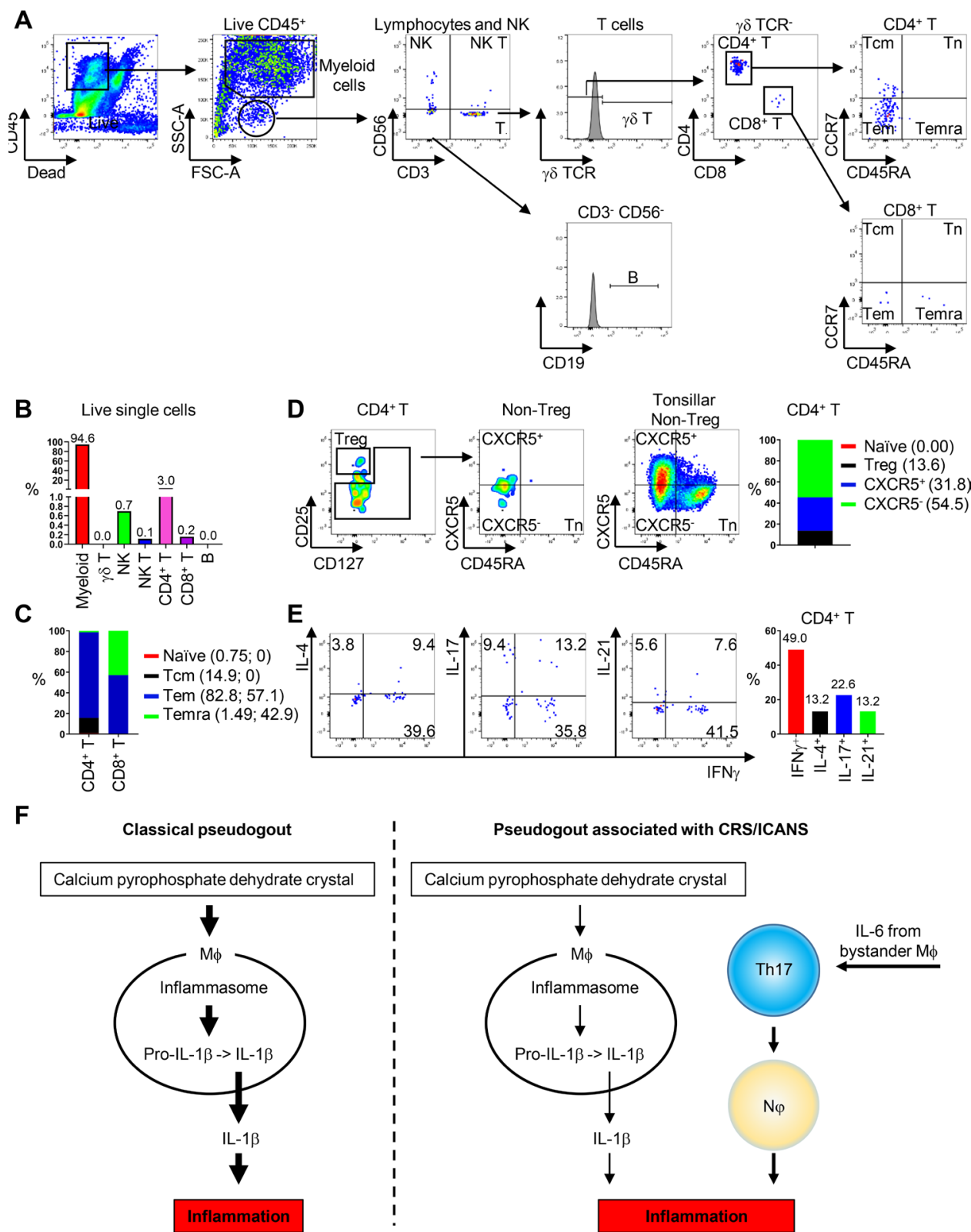

Figure 1 Flow cytometry analysis of synovial immune cells at pseudogout arthritis flare. (A) Flow cytometry gating strategy of major immune cells. Temra, terminally differentiated T cells. (B) Percentage of major immune cell subsets within total live single cells. (C) Percentage of T cell subsets. (D) Percentage of $\mathrm{CD}^{+}{ }^{+} \mathrm{T}$ cell subsets. Flow cytometry plots of $\mathrm{CD} 4^{+} \mathrm{T}$ cells and quantitative analysis. Gating of CD45RA and CXCR5 was made based on the expression of CD45RA and CXCR5 on anonymous tonsillar non-Tregs. (E) Percentage of cytokine-producing $\mathrm{CD} 4^{+} \mathrm{T}$ cells. Flow cytometry plots and quantitative analysis. (F) Hypothetical model of pseudogout arthritis associated with CRS/ICANS. M $\varphi$, macrophages; $N \phi$, neutrophils. CRS, cytokine release syndrome; CXCR5, C-X-C chemokine receptor type 5; FSC-A, forward scatter area; ICANS, immune effector cell-associated neurotoxicity syndrome; IFN $\gamma$, interferon- $\gamma$; IL-17, interleukin 17; NK, natural killer cells; NK T, NK T cells; SSC-A, side scatter area; TEM, effector memory T cells; TCM, central memory T cells; TN, naïve T cells; Treg, regulatory T cells.

Combo 2, Meso Scale Discovery), according to the manufacturer's instructions.

\section{RESULTS}

Flow cytometry revealed that myeloid cells were most dominant major immune cell subsets in the synovial fluid $(94.6 \%$ within live single cells) (figure 1A,B). The effector memory population was increased within both $\mathrm{CD}^{+}$and $\mathrm{CD}^{+} \mathrm{T}$ cells, suggesting they may be involved in the pathogenesis of the pseudogout arthritis in our case (figure 1C).

Given the central role of $\mathrm{CD} 4^{+} \mathrm{T}$ cells in immune responses, we focused on $\mathrm{CD} 4^{+} \mathrm{T}$ cells (figure $1 \mathrm{D}, \mathrm{E}$ ). The most abundant $\mathrm{CD} 4^{+} \mathrm{T}$ cell subset was CXCR5 $5^{-}$ $\mathrm{CD}^{+}$effector $\mathrm{T}$ cells $\left(54.5 \%\right.$ within live $\mathrm{CD} 4^{+} \mathrm{T}$ cells) (figure 1D). Intracellular staining of $\mathrm{CD} 4^{+} \mathrm{T}$ cells showed predominance of IFN $\gamma$ producing $\mathrm{CD}^{+} \mathrm{T}$ helper 1 (Th1) cells and IL-17 producing CD4 $4^{+} \mathrm{T}$ helper 17 (Th17) cells 


\begin{tabular}{lc} 
Table 2 & Cytokine concentration in synovial fluid \\
\hline Cytokines & Concentration $\mathbf{( p g / m L )}$ \\
\hline IFN $\gamma$ & 5.41 \\
TNF $\alpha$ & 2.69 \\
GM-CSF & 0 \\
IL-1 $\beta$ & 30.26 \\
IL-2 & 4.64 \\
IL-4 & 0.10 \\
IL-6 & 14227.73 \\
IL-10 & 2.50 \\
IL-17A & 1.69 \\
IL-21 & 16.02 \\
IL-22 & 0.62 \\
\hline
\end{tabular}

GM-CSF, granulocyte-macrophage colony-stimulating factor; IFN $\gamma$, interferon $\gamma$; IL-2, interleukin 2; TNF $\alpha$, tumor necrosis factor $\alpha$.

$\left(49.0 \%\right.$ and $22.6 \%$ within $\mathrm{CD}^{+} \mathrm{T}$ cells, respectively) (figure 1E). Multiplex analysis of the SF supernatant revealed the significant levels of IL-6, a key cytokines for Th17 cell differentiation, function, and plasticity (table 2). ${ }^{9}$

\section{DISCUSSION}

To the best of our knowledge, this is the first report of a case of newly developed, crystal-proven, pseudogout arthritis associated with CRS/ICANS after cell therapy. Of note, the patient's pseudogout flare was resolved with tocilizumab. Our clinical observation is particularly important as steroids, the first-line therapy for pseudogout arthritis, are frequently avoided for the treatment of IEC-associated toxicities due to their potential lymphocytolytic activity. ${ }^{13}{ }^{310}$ Immunoprofile of synovial fluid revealed the dominance of Th1 and Th17 cells with abundant IL-6.

Innate immune cells, primarily neutrophils and macrophages, are pivotal in the pathogenesis of pseudogout arthritis. Macrophages identify and phagocytose CPPD crystals, activating Nod-like receptor protein inflammasomes, with subsequent secretion of IL-1 $\beta$, a potent inflammatory cytokine. ${ }^{11}$ In CRS, inflammatory cytokines secreted from the CAR-T cells can activate bystander immune cells, including macrophages, monocytes, and dendritic cells, with subsequent production of IL- $6{ }^{12}$ IL-6 is known to be important for the differentiation, function, and survival of Th17 cells, which are a subset of $\mathrm{CD}^{+}{ }^{+} \mathrm{T}$ cells involved in many autoimmune diseases. ${ }^{89}$ Interestingly, the proportion of Th17 cells in the synovial fluid in our case was substantially higher than what has been previously reported in classical inflammatory arthritis. ${ }^{13}$ Thus, it is plausible that, in our case, inflammatory cytokines activated bystander macrophages, which secreted IL-6, thus contributing to Th17 differentiation, function, and survival. In turn, these Th17 cells enhanced recruitment of neutrophils to the site of the CPPD crystal deposition. Taken together, we speculate that in addition to IL-1 $\beta$ induced by the CPPD crystal, an IL-6-Th17-neutrophil axis played a critical role in the pathogenesis of CPPD arthritis in our patient. This hypothesis is supported by the clinical observation that the patient's pseudogout arthritis was resolved completely with tocilizumab. We show our hypothesis graphically in the figure $1 \mathrm{~F}$.

This is the first case reporting pseudogout arthritis in a patient who developed CRS and ICANS after T cell therapy. Importantly, pseudogout arthritis of our patient was resolved with tocilizumab. Because IEC therapy is being increasingly used in various cancers, there may be an increased recognition of CPPD arthritis in patients undergoing IEC therapy, and clinicians, including hematologists, oncologists, and rheumatologists, should be aware that IEC might be associated with the development of arthritis. Immunophenotypic analyses in our case suggest the contribution of a feed-forward loop between macrophages, neutrophils, and Th17 cells in the joint inflammation. Comprehensive analyses of more cases and control samples are warranted to understand the underlying mechanisms of pseudogout arthritis and its potential association with IEC therapy.

Contributors JT, SF and DSH. managed the case; STK. performed experiments, analyzed the data, and wrote the manuscript; DK, EN, AS and JM. analyzed and discussed the data; all authors reviewed and edited the manuscript.

Funding This study was supported by the University of Texas MD Anderson Cancer Center Division of Internal Medicine Bridge Fund (STK).

Competing interests EN, AS and JM are employees of Adaptimmune LLC and own stock in the company. DSH has research fundings from AbbVie, Adaptimmune, Adlai Nortye, Amgen, Astra-Zeneca, Bayer, Bristol Myers Squibb, Daiichi-Sankyo, Eisai, Eli Lilly, EMD Serono, Erasca, Fate Therapeutics, Genentech, Genmab, GlaxoSmithKline, Ignyta, Infinity, Kite, Kyowa, LOXO, Merck, Medlmmune, Millenium, Mirati, miRNA, Molecular Templates, Mologen, Navier, NCI-CTEP, Novartis, Numab, Pfizer, Seattle Genetics, Takeda, Turning Point Therapeutics, Verstatem, and VM Oncology. DSH has supports for travel, accommodations, and expenses from AACR, Amgen, ASCO, Astra Zeneca, Bayer, Celgene, Eli Lilly, Genentech, Genmab, GlaxoSmithKline, Janssen, LOXO, miRNA, Pfizer, Philips, SITC, and Takeda. DSH declares roles as a consultant, speaker, or advisor for Alpha Insights, Acuta, Amgen, Axiom, Adaptimmune, Baxter, Bayer, Boxer Capital, COG, Ecor1, Genentech, GLG, Group H, Guidepoint, HCW Precision, Infinity, Janssen, Merrimack, Medscape, Numab, Pfizer, Prime Oncology, Seattle Genetics, ST Cube, Takeda, Tavistock, Trieza Therapeutics, and WebMD. DSH. has other ownership interests with Molecular Match (Advisor), OncoResponse (Founder) and Presagia (Advisor).

Patient consent for publication Not required.

Ethics approval Synovial fluid collection for the study was approved by an Institutional Review Board at The University of Texas MD Anderson Cancer Center (IRB \#: PA16-0935). Consent to participate was obtained.

Provenance and peer review Not commissioned; externally peer reviewed.

Open access This is an open access article distributed in accordance with the Creative Commons Attribution Non Commercial (CC BY-NC 4.0) license, which permits others to distribute, remix, adapt, build upon this work non-commercially, and license their derivative works on different terms, provided the original work is properly cited, appropriate credit is given, any changes made indicated, and the use is non-commercial. See http://creativecommons.org/licenses/by-nc/4.0/.

\section{REFERENCES}

1 Maus MV, Alexander S, Bishop MR, et al. Society for immunotherapy of cancer (SITC) clinical practice guideline on immune effector cellrelated adverse events. J Immunother Cancer 2020;8:e001511. 
2 Brudno JN, Kochenderfer JN. Recent advances in car T-cell toxicity: mechanisms, manifestations and management. Blood Rev 2019;34:45-55

3 Dholaria BR, Bachmeier CA, Locke F. Mechanisms and management of chimeric antigen receptor T-cell therapy-related toxicities. BioDrugs 2019;33:45-60.

4 Neelapu SS. Managing the toxicities of car T-cell therapy. Hematol Oncol 2019;37 Suppl 1:48-52

5 Martinon F. Mechanisms of uric acid crystal-mediated autoinflammation. Immunol Rev 2010;233:218-32.

6 Richette P, Bardin T, Doherty M. An update on the epidemiology of calcium pyrophosphate dihydrate crystal deposition disease. Rheumatology 2009;48:711-5.

7 Kim ST, Bittar M, Kim HJ, et al. Recurrent pseudogout after therapy with immune checkpoint inhibitors: a case report with immunoprofiling of synovial fluid at each flare. J Immunother Cancer 2019;7:126.

8 Craft JE. Follicular helper T cells in immunity and systemic autoimmunity. Nat Rev Rheumatol 2012;8:337-47.
9 Harbour SN, DiToro DF, Witte SJ, et al. $\mathrm{T}_{H} 17$ cells require ongoing classic IL-6 receptor signaling to retain transcriptional and functional identity. Sci Immunol 2020;5. doi:10.1126/sciimmunol.aaw2262. [Epub ahead of print: 17 Jul 2020].

10 Strati P, Ahmed S, Furqan F, et al. Prognostic impact of corticosteroids on efficacy of chimeric antigen receptor T-cell therapy in large B-cell lymphoma. Blood 2021. doi:10.1182/ blood.2020008865. [Epub ahead of print: 03 Feb 2021].

11 Eisenbarth SC, Colegio OR, O'Connor W, et al. Crucial role for the NALP3 inflammasome in the immunostimulatory properties of aluminium adjuvants. Nature 2008;453:1122-6.

12 Santomasso B, Bachier C, Westin J, et al. The other side of car T-cell therapy: cytokine release syndrome, neurologic toxicity, and financial burden. Am Soc Clin Oncol Educ Book 2019;39:433-44.

13 Zizzo G, De Santis M, Bosello SL, et al. Synovial fluid-derived T helper 17 cells correlate with inflammatory activity in arthritis, irrespectively of diagnosis. Clin Immunol 2011;138:107-16. 